\title{
Is it an orbital foreign body?
}

\author{
Mohamed Shaheeda, Stacey C Lam *, Noel CY Chan, Hunter KL Yuen
}

Hong Kong Med J 2017;23:653.e1-2

DOI: $10.12809 / \mathrm{hkmj} 164952$

A 58-year-old man presented with left globe rupture following blunt orbital trauma. Visual acuity was light perception only. There was lid swelling, ecchymosis, and a deformed globe with scleral laceration. Computed tomography $(\mathrm{CT})$ of the orbit revealed a $3.7-\mathrm{mm}$ hyperdensity at the superomedial aspect of the left globe, interpreted by the radiologist as a possible intra-orbital foreign body (Fig).

Thorough history for the mechanism of trauma, physical examination for a wound site and thin cut CT sections were reviewed. History revealed blunt orbital trauma by a metallic rod rather than a sharp penetrating injury. No cutaneous entry site was evident. Computed tomography revealed bilateral hyperdensities at the level of the trochlea, more pronounced on the left than the right, with no metallic streak artefacts. All of the above supported a diagnosis of trochlear calcification rather than an intra-orbital foreign body, and the patient subsequently underwent emergency repair of scleral laceration without orbital exploration.

\section{Discussion}

The trochlea is a cartilaginous pulley at the superomedial aspect of the orbit through which the superior oblique muscle tendon passes freely. It has a synovium-lined space, and like other synovial joints of the body, the trochlea can develop calcifications in

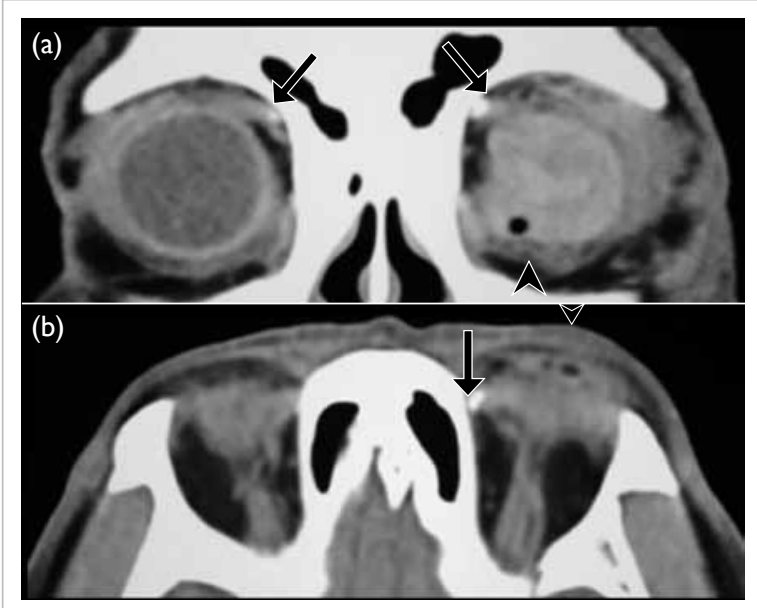

FIG. (a) Coronal computed tomographic scan in soft tissue window showing bilateral hyperdensities at the superomedial aspect of both orbits at the level of the trochlear apparatus (arrows). There is also a left collapsed globe with intra-ocular haemorrhage and gas (arrowheads). (b) Axial computed tomographic scan in soft tissue window showing hyperdensity at the anteromedial left orbit (arrow) the cartilage, tendon, or within the bursa-like cleft. ${ }^{1}$ The reported prevalence of incidental trochlear calcifications on CT is $3-16 \%$, with over $50 \%$ being unilateral., ${ }^{2,3}$ No clear cause has been identified, but prior studies have postulated degenerative, inflammatory, metabolic or traumatic aetiologies. ${ }^{4}$ Since trochlear calcification is asymptomatic, most cases go unnoticed by radiologists as well as ophthalmologists. In the presence of co-existing orbital trauma, it can be misdiagnosed as an intraorbital foreign body. Surgical exploration around the trochlear region may cause damage and scarring leading to diplopia.

Differentiation of the two entities requires history taking, physical examination, and proper imaging. A foreign body is present in one in six cases of orbital trauma, with metallic objects and glass being the most common. ${ }^{5}$ A review of the history to determine mechanism of injury is useful but may be unreliable. Examination of the skin and conjunctiva, particularly the fornices, may help detect subtle penetrating injuries. Radiological assessment includes plain films, ultrasound, CT, and magnetic resonance imaging. Plain films are limited to detection of metallic foreign bodies, and ultrasound has limited use because foreign bodies can be masked by surrounding highly reflective structures such as bone. Computed tomography is an excellent means to identify high-density objects such as metal or glass, but not organic matter. Magnetic resonance imaging is contra-indicated in metallic foreign bodies, but may be useful for organic foreign matter. Features of trochlear calcification may include symmetrical presentation and typical site at the trochlear apparatus. Its morphology has been described as comma, dot, and inverted U shape. ${ }^{2}$ In contrast, orbital foreign bodies are often unilateral with no specific size, shape, or location. Metallic foreign bodies may also generate streak artefacts.

Orbital calcifications can be incidental or pathological. It is important to recognise trochlear calcifications as distinct from foreign bodies so as to avoid unnecessary surgical exploration in the presence of orbital trauma.

M Shaheeda, FRCSEd, MPH

SC Lam *, MB, ChB

NCY Chan, FRCSEd, FCOphthHK

HKL Yuen, FRCSEd, FCOphthHK

Department of Ophthalmology and Visual Sciences, The Chinese University of Hong Kong, Shatin, Hong Kong

* Corresponding author: staceylam@gmail.com 


\section{References}

1. Sobel RK, Goldstein SM. Trochlear calcification: A common entity. Orbit 2012;31:94-6.

2. Xiao TL, Kalariya NM, Yan ZH, et al. Trochlear calcification and intraorbital foreign body in ocular trauma patients. Chin J Traumatol 2009;12:210-3.

3. Shriver EM, McKeown CA, Johnson TE. Trochlear calcification mimicking an orbital foreign body. Ophthal Plast Reconstr Surg 2011;27:143-4.

4. Buch K, Nadgir RN, Tannenbaum AD, Ozonoff A, Fujita A, Sakai O. Clinical significance of trochlear calcifications in the orbit. AJNR Am J Neuroradiol 2014;35:573-7.

5. Nasr AM, Haik BG, Fleming JC, Al-Hussain HM, Karcioglu ZA. Penetrating orbital injury with organic foreign bodies. Ophthalmology 1999;106:523-32. 